\title{
EL AMBIENTE Y LA CULTURA: CAMPOS DE PROBLEMATIZACIÓN DE LAS CIENCIAS AMBIENTALES Y LA EDUCACIÓN
}

\author{
Environment and Culture: \\ Problematization fields of environmental science and education \\ LUIS ANTONIO GONZÁLEZ 1 \\ 1Universidad del Valle
}

E-mail: lage49@yahoo.es

\section{Resumen}

Un objetivo de las ciencias ambientales es entender la relación naturaleza, hombre, y sociedad. La educación es un proceso interpersonal que permite comprender la situación problemática del ambiente. En este artículo tomamos como referencia los humedales tipo Cauces de río abandonado (CRA), para ir valorando la práctica de la técnica educativa de escenarios comparados (TEDEC), en la aplicación de las ciencias ambientales. Hacemos relevante la responsabilidad de los educadores en los procesos de identificación de factores y el manejo de la observación organizada, para ir integrando los componentes de las ciencias sociales con fundamento en la economía política y las ciencias ecológicas. De esta manera se quieren fortalecer las acciones de pensamiento, la profundización en el conocimiento, la percepción que se adquiere de la realidad, de las prácticas productivas y culturales, en las transformaciones y repercusiones que éstas puedan tener en el ambiente.

Palabras claves: Ambiente, Cultura, Ciencias Ambientales, Educación, Realidad.

\section{Abstract}

One objective of environmental science is to comprehend the relationship between nature, man and society. Education is an interpersonal process that allows us to understand the problematic situation of the environment. In the article, we take as reference, abandoned rivers channels in order to evaluate the practice of educational techniques in comparative scenarios, on the application of environmental sciences. We make relevant the responsibility of educators in the process of identifying factors and the management of organized observation, to integrate the components of the social sciences grounded in political economy and ecological sciences. This way, we want to strengthen the actions of thought, the deepening in the knowledge, the perception taken from reality, from productive and cultural practices, in the transformations and impacts that may have in the environment.

Keywords: Environment, Culture, Environmental Science, Education, Reality. 


\section{INTRODUCCIÓN}

El presente ensayo dentro del marco de la relación naturaleza, cultura y sociedad, propone algunas reflexiones orientadas a la comprensión del papel de las ciencias ambientales en las transformaciones del mundo, es decir de la naturaleza, la sociedad y el pensamiento. En este sentido vale la pena preguntarse: ¿Cuál es el papel de las ciencias ambientales en la relación existente entre sociedad, naturaleza y cultura?, ¿En qué sentido las ciencias ambientales en la cultura occidental, con una forma de saber, es capaz de integrar a las ciencias naturales y sociales?

Las ciencias ambientales como cualquier otra forma de cultura determinada que organiza un saber, se construye a través de un lenguaje que le es propio, mediante el cual el ser humano formula su relación con la naturaleza, consigo mismo, con la cultura, la sociedad, con su ambiente y la humanidad. En otras palabras, la ciencia ambiental pretende generar una versión científica que confronta otras ciencias que tienen que ver con la naturaleza, el comportamiento, la conducta, la responsabilidad, etc., y desde esta visión es que el ser humano se cuestiona a sí mismo y su relación con el ambiente, en el marco de una nación como Colombia biológica, ecológica y culturalmente diversa.

Finalmente este tipo de pensamiento que profundiza en la relación entre ciencias ambientales y educación como acción humana, permite definir un conjunto de orientaciones que en el ideal de formación de sujetos, propone nuevos sentidos a la gramática formativa que incorpore al núcleo de su interés tanto el sujeto histórico como la ciencia inter y transdisciplinar, al ethos ecoresponsable, al ethos transcultural y al ethos planetario.

Las ciencias ambientales; una forma de cultura para comprender la relación naturaleza-hombre-sociedad

El interés por las ciencias ambientales está determinado más por necesidades prácticas reales de la vida moderna, deterioro ecológico, degradación de la vida y por exigencias cognoscitivas, que por simples caprichos esnobistas. El objetivo de conocimiento de las ciencias ambientales es la naturaleza planetaria en su conjunto, vale decir el sistema integral que forman los organismos con el ambiente donde se desarrollan. Desde la antigüedad se precisó la trascendencia de esta estrecha relación dialéctica del hombre y la naturaleza, pero al considerar la cultura, la sociedad, la economía, la política y el desarrollo, conformó el componente más propiamente humano de las ciencias ambientales.

Los problemas y las problemáticas ambientales están ligados a la transformación y el dominio de la naturaleza, los cuales se han acentuado considerablemente, poniéndose de presente las temáticas relacionadas con las ciencias ambientales, las cuales son necesarias para trabajar conjuntamente con enfoques teóricos y metodologías que permitan acceder a la complejidad ambiental. Asumiendo una postura epistemológica que garantice abordar la integración de las ciencias naturales y sociales, desde una perspectiva interdisciplinaria y transdisciplinaria, y como una nueva área del conocimiento como desafío a la complejidad ambiental. La problemática ambiental plantea la necesidad de internalizar un saber ambiental emergente en todo un conjunto de disciplinas para construir un conocimiento capaz de captar la multicausalidad y las relaciones de interdependencia de los procesos del orden natural y social, así como para construir un saber y una racionalidad social orientados hacia objetivos de un desarrollo sustentable equitativo y duradero (Cubillos 2007, Duque 2007, Leff 2007, Noguera 2007).

Las preguntas que frecuentemente se pueden plantear desde las ciencias ambientales, no encuentran respuestas suficientes y completas a través de los métodos utilizados en las ciencias naturales. Es por esto que para desarrollar un trabajo en ciencias ambientales, sin desconocer el valioso aporte de las ciencias naturales, se requiere también, tener en cuenta las herramientas teóricas y metodológicas proporcionadas por las ciencias sociales y humanas. Desde la Antropología y la economía política es importante analizar las prácticas culturales y los estilos de desarrollo que actúan sobre la base natural para comprender los procesos de transformación en los territorios. La Economía política que enfatiza en lo ambiental, desde el punto de vista teórico, toma como fuente de análisis el modelo de interacción Naturaleza- Cultura -Sociedad, y a nivel práctico se fundamenta en los modelos de desarrollo que realizan las sociedades sobre sus territorios, presionando o manejando adecuadamente la base natural.

Es una perspectiva que posibilita plantear; que la principal contribución de la economía política a la dimensión ambiental consiste en su manera de ver lo real y la realidad.

Desde esta perspectiva, la capacidad para entender que el pensamiento integral y no fragmentado, no es una sumatoria de disciplinas sino la capacidad de poner a circular el conocimiento de manera interdisciplinaria y transdisciplinaria, que está en el pensamiento mismo y en el acto de pensar para proyectarlo hacia la realidad. De esta manera la capacidad de reconocer el conjunto de circunstancias en el espacio donde nos movemos a través de nuestra manera de pensar nos permite ser conscientes de lo que pasa, lo que ocurre y poder tener la capacidad de 
organizar con un sentido que responda a las necesidades del momento del pensamiento para dar cuenta de aquello que no conozco pero que me determina de alguna manera (González 2006).

\section{En Colombia ha existido la tendencia a ignorar la existencia de las etnias}

Las diversas culturas en Colombia llegaron a una relativa consolidación de las estrategias de crecimiento y prosperidad que desarrollaron y aplicaron con éxito a un ámbito de la extensión vertical y horizontal ecológica y a la complejidad en sus niveles de pensamiento. De esta manera habían logrado la coherencia entre territorio, ecología, población, idioma, cultura, formas de organización social del trabajo, la propiedad y el gobierno. Con la conquista y la irrupción de los conquistadores europeos se detuvo este proceso de consolidación cultural que había sido heredado hace miles de años, ocasionando su degradación con la consecuente desarticulación de sus niveles ecológicos, los genocidios y la desaparición de las culturas étnicas. A nivel ecológico y social, la constatación del punto anterior conducía a la certidumbre de que la sobrevivencia individual era imposible en la hostilidad de los distintos pisos térmicos de los distintos territorios y aún muy dificultosa en los lugares más favorecidos, que habitaban en la verticalidad de los mismos. En consecuencia, la organización social sólo podía estar basada en una intensa cooperación colectiva, sustentada en profundos lazos familiares, comunales, étnicos e interétnicos en ese orden, y la propiedad comunitaria sobre la tierra (Portela 2012).

Los estudios antropológicos y arqueológicos a nivel mundial, reconocen que la manera como abordan las distintas funciones de las poblaciones humanas asentadas, revelan un tipo de prácticas culturales como las de manejo del agua, adaptación a las variaciones climáticas, manejo de territorios agrestes de páramo, etc., lo hacen a partir de un saber que refleja el conocimiento de las leyes ecológicas en especial las de conservación, aumentando la productividad de su entorno al seleccionar y domesticar los alimentos. Su desarrollo social está muy ligado al desarrollo de las relaciones sociales específicas en los espacios socioproductivos. En efecto, la fabricación y utilización del más simple instrumento, la herramienta de piedra, sólo pudo realizarse en la medida en que los seres humanos establecieron relaciones sociales específicas de producción y convivencia (Rodríguez 2012).

El desarrollo antropológico se caracterizó por la incorporación gradual de la ecología a la economía de aquí que el progreso del hombre esté ligado a la actividad productiva. Frente a esto hay que tener en cuenta la manera como se logran estas transformaciones que afectan la estabilidad natural y sus regulaciones.

El concepto de territorio existe desde el mismo momento en que el ser humano hizo posible el uso del suelo, marcando el territorio por defensa propia y subsistencia. De esta manera, humaniza el territorio a través de su inteligencia y desarrolla un pensamiento para la convivencia, el respeto por el territorio y propone estrategias para su transformación (García 1976). La conceptualización territorial complejiza la construcción colectiva hacia la conservación y recuperación de tradiciones, saberes populares, vivencias cotidianas y patrones culturales, donde los diferentes sistemas de conocimiento tienen profundos significados y se constituyen en medios de aprendizaje y de adaptación para la comunidad.

Desde esta perspectiva los estudios en las ciencias Ambientales tienen como estrategia estudiar las respuestas que los grupos humanos de manera cultural generan frente a los problemas y problemáticas ambientales. De esta manera, las ciencias ambientales valoran y acogen el conocimiento significativo de las distintas ciencias frente a los problemas ambientales concretos, contribuyendo a identificar modos de vida y prácticas sustentables en el seno de las comunidades, no sólo desde el actuar en la relación cultura-ecosistema sino desde el punto de vista cultural, social, político y humano, al relacionarlos con el tipo de cosmovisión, valores, creencias, estructuras de parentesco, tradiciones, rituales, pensamiento, ideologías políticas y su relación con la política de humanidad y vida. Desde este punto de vista la problemática antropológica se enmarca dentro de las organizaciones sociales y los impactos de los diferentes estilos de desarrollo y manejo de los recursos (Descola \& Palsson 2001).

\section{La relación entre Ciencias ambientales y sociedad}

Las actividades características del ser humano y de manera especial los procesos productivos, que constituyen la base de la riqueza y el progreso social, deben considerarse regidas fundamentalmente por principios económicos, históricos, políticos y culturales y no integrando de manera mecánica a la sociedad humana, las leyes propias de las poblaciones biológicas, que generan determinismos absolutistas. Desde esta perspectiva de integración dialéctica recíproca de lo natural y lo social, surgen aspectos que se contraponen y se identifican a la vez pero donde el aspecto social juega el papel determinante. La presencia del ser humano en los sistemas ecológicos debido a la consecución de sus medios de vida, está en estrecha relación con su capacidad para transformar conscientemente la naturaleza, la sociedad y el pensamiento. En el ámbito de las ciencias 
ecológicas y por lo tanto de las ciencias ambientales, está relacionado al componente natural de la actividad productiva que no pueden concebirse más, que por medio de una organización económica, social, política y cultural, muy superior y diferenciable cualitativamente de los sistemas biológicos.

Se necesita establecer y conocer las conexiones entre ambiente, estilos de desarrollo y orden económico mundial, nacional, regional y local, desde una perspectiva del conflicto social, político y de los movimientos sociales. Los procesos de destrucción ecológica y degradación socioambiental han sido resultado de prácticas inadecuadas de uso del suelo, que dependen de patrones tecnológicos y de un modelo agresivo de crecimiento y producción que permiten maximizar ganancias económicas en el corto plazo, revirtiendo sus costos sobre los sistemas naturales y sociales manera de separar la relación hombre naturaleza imponiéndose el interés económico (Naredo 1993).

Por eso es imprescindible una adecuada planificación del territorio que permita cumplir con el ordenamiento territorial, la Intervención interinstitucional articulada con programas de educación para la sustentabilidad y de actividades productivas de bajo impacto, evitando las imprevisiones estatales en el manejo de los recursos en especial el manejo del agua y la energía. En este sentido se puede decir que la esencia actual de la crisis del agua radica en el manejo inadecuado de las cuencas y, su solución dependerá de la superación de las barreras políticas que limitan la explotación nacional del recurso y un esfuerzo investigativo intenso sobre la naturaleza de el mismo. Por esto la estrategia para atender este problema debe mirarse más como una manifestación de problemas políticos, sociales y económicos, que de tipo técnico. Desde las ciencias ambientales es importante considerar aspectos como la equidad y la participación comunitaria pues resultan imprescindibles en el análisis de la gobernanza que relaciona aspectos de la gestión y distribución de los recursos y la influencia de ciertos grupos de poder o clases sociales (Meerganz 2006).

Como la crisis ambiental y los problemas están ligados a las transformaciones del planeta, como el calentamiento global, el efecto invernadero, la lluvia ácida, el estrés nuclear, la extinción de la biodiversidad, etc., y como esto depende de la estrategia relacionada entre la ecología, la economía, lo social y la política, se establecen muchas interpretaciones, ideologías y filosofías, entremezclados con intereses sociales y de clases que se encuentran implicados en la situación problemática. Desde este punto de vista las ciencias ambientales analizan los múltiples factores que se encuentran implicados en la situación ambiental $\mathrm{y}$, aparentemente es cierto que el hombre puede verse como el causante del problema, algo parecido se entiende también para el progreso, el avance científico y tecnológico, ingeniería genética, productos transgénicos, la energía nuclear, etc. Un análisis más detallado nos precisa lo contrario, es el estancamiento, los bajos niveles de organización social, la falta de humanización, el fracaso social, la verdadera causa de los problemas ambientales en los que el ser humano al igual que en la tecnología se convierten en el distractor político implicado en la situación problema.

Cuando hay evolución social y cultural, cuando se transforma la sociedad y la manera de pensar, a partir del cambio en la actividad manual e intelectual en el trabajo, entonces se alcanza la fase de organización social que permite el éxito, logrando beneficios para una comunidad, que representan la mayoría y el todo.

Esto obliga a una reestructuración económica, tecnológica y social, pero, ante todo, una reorientación cultural que supere los antropocentrismos, y genere el espacio para dar lugar a una nueva conciencia humana que conlleve a una ética basada en:

- La valoración de las culturas y los recursos naturales de cada región.

- La sostenibilidad ecológica y el mejoramiento de la calidad de vida.

- El desarrollo de los niveles de organización social.

- El respeto por la naturaleza y la vida.

- La participación comunitaria para llevar a cabo la concertación previa y posteriormente la gestión comunitaria.

\section{Educación; un acto de interpersonalidad en devenir de la interculturalidad}

La Constitución Política de Colombia de 1991 reconoce la diversidad étnica y cultural de la nacionalidad, lo cual conlleva a una implicación pedagógica que se concreta en la necesidad de una educación en devenir de la interculturalidad, no solamente para los pueblos culturalmente diferenciados, sino también para la sociedad nacional colombiana, que tiene el deber y el derecho de valorar nuestras culturas, en una dimensión de alteridad cultural a partir de un diálogo respetuoso de saberes, de la tolerancia y la circulación de los conocimientos que se articulan y se complementen mutuamente, valorando su propia forma de pensar y organizar su mundo.

La educación se hace desde la cultura, a través de las comunidades se propende por formar personas cada vez 
más autónomas y participativas en el sentido de que los principios, fundamentos, objetivos, y funciones sean diseñados por las mismas comunidades, plasmando en ello el proyecto de vida que articula en el sentir del devenir histórico de ese pueblo, sus decisiones de tipo colectivo que interesan e involucran a todos, y en las cuales es preciso evitar los riesgos de degradación para la vida, la cultura, la ecología y el ambiente. Es necesario analizar profundamente el papel del maestro o del agente educativo dentro de los procesos educativos, su capacitación y formación, su perfil, sus relaciones y compromisos con los estudiantes, con la comunidad, con sus autoridades y organizaciones, con las instituciones, con el desarrollo local, regional y nacional. Se requiere que en la comunidad educativa se discutan nociones y conceptos como los de educación, cultura, seres humanos, sociedad, escuela, democracia, autonomía, humanidad, política de vida, etc., puesto que en la misión de la institución escolar se define, precisamente la relación de estos conceptos con el mundo y la vida. En este contexto la escuela se convierte en un mecanismo propio de socialización y creación de procesos, en un foro de crecimiento, prosperidad y construcción de sentido de vida, y en un espacio de sistematización, construcción, formalización, ampliación, valoración, recuperación y producción de conocimientos y saberes. Hay una alerta sobre la necesidad de potenciar al estudiante como persona en todas su facultades, lo que requiere pensamiento, creatividad, tener conciencia de los problemas y su problemática ambiental, donde se dé la potenciación para desarrollar lo histórico, los mecanismos psicológicos, la comunicación, etc., es todo un desafío de formación para que estén a la altura de los desafíos del momento actual (González 2006).

\section{Campos de conocimiento en las ciencias ambientales desde la época presente}

Al caracterizar el mundo como un todo dinámico cambiante, como un conjunto de realidades cuyos bordes de carácter abierto, variable y potencial admiten, que la razón humana pueda encontrar modos de intelección y de comprensión de esos dominios y órdenes de realidad con la dinámica compleja que los caracteriza; reconociendo también las transformaciones sustanciales en las gramáticas de intervención y elaboración de conocimiento de esos órdenes $\mathrm{y}$ dominios emergentes de realidad.

Las formales maneras de trabajo humano con el conocimiento ambiental y planetario están en permanente proceso de autocomprensión; ello significa que a la vez que se reconoce una movilidad en las lógicas y las epistemologías de las formaciones disciplinares, se reconocen también ensambles innovadores entre las formas de actuación de la humana racionalidad; es en su territorio, en el de la razón constitutivas de los modos de conocimiento que fundan la sabiduría patrimonial de la humanidad, en el cual se producen los más interesantes movimientos.

Desde las ciencias ambientales se hace posible capturar nuevas áreas de trabajo, nuevos intereses, nuevos campos, nuevos interrogantes, nuevas expectaciones. Entre los nuevos conocimientos resultantes del esfuerzo inter, poli y sobre todo transdisciplinar de conocimiento que se están empezando a circular dentro y por entre espacios colegiados de la asociaciones humanas que se relacionan con la tierra, la sociedad, la cultura, la vida, la coexistencia y la humanidad, creando nodos categoriales como el de planetariedad, tierra-patria, destino-obra de vida, comunidad de destino, pragma- obra de humanidad, política de civilización, política de vida, los cuales se constituyen en la base para la construcción de planos de interrogación en torno a la era planetaria y sus marcas de la edad de hierro, de los linderos de la política de la vida, de los dominios del hombre, de la política de inclusión generalizada de la existencia y de la política para la estética de la vida (Morin 1999).

\section{REFERENCIAS}

CUBILLOS, L. F. 2007. La epistemología de las ciencias ambientales: reflexiones desde la "impertinencia" social. Tomado de la Red Colombiana de Formación Ambiental, RCFA. Las ciencias ambientales: Una nueva área de conocimiento. Bogotá, D.C. 182 pp.

DESCOLA, P. \& PALSSON, G. 2001. Naturaleza y sociedad: perspectivas antropológicas. Construyendo naturalezas. SIGLO XXI editores S. A., México. 359 pp.

DUQUE, A. A. 2007. Ambiente como categoría y ciencia como conocimiento. Tomado de la Red Colombiana de Formación Ambiental, RCFA. Las ciencias ambientales: Una nueva área de conocimiento. Bogotá, D.C.

GARCIA, J. L. 1976. Antropología del territorio. Taller de Ediciones Josefina Betancur. Madrid.

GONZÁLEZ, L. A. 2006. Pedagogía y estrategias de educación ambiental. Editorial Poemia. Cali Valle Colombia.

LEFF, E. 2007. La complejidad ambiental: del logos científico al diálogo de saberes. Tomado de la Red Colombiana de Formación Ambiental, RCFA. Las ciencias ambientales: Una nueva área de conocimiento. Primera edición, diciembre de 2007. Bogotá, D.C. Colombia. 
MEERGANZ, V. M. G. 2006. Flujos de agua, flujos de poder. La aportación de Erik Swyngedouw al debate sobre los recursos hídricos en latinoamérica y en el estado español. Documents d'anàlisi geogràfica. $N^{\circ} 47,2006$, págs. 129-139 DOI:(Revista) Universidad de Barcelona.

NAREDO, J. M. 1993. Historia de las relaciones entre economía, cultura y naturaleza. En: PEÑA, F. G. (ed). pp. Introducción a la Ecología Política: 57-97. Comares Editorial, Granada.

MORIN, E. 1999. L'intelligence de la complexite. L'Harmattan, París. 332 pp.

NOGUERA, A. P. 2007. Emergencia de una episteme-éticoestética-política que constituye un nuevo concepto de ciencia desde el pensamiento ambiental complejo. Tomado de la Red Colombiana de Formación Ambiental, RCFA. Las ciencias ambientales: Una nueva área de conocimiento. Primera edición, diciembre de 2007. Bogotá, D.C.

PORTELA, H. 2012. La naturaleza y la cultura en la urdimbre de las cosmovisiones que sustentan la vida. Pacífico colombiano. Conferencia en el Programa doctorado interuniversitario de Ciencias ambientales. Universidad del Cauca. Colombia.

RODRÍGUEZ, C. A. 2012. Evidencias arqueológicas sobre la agricultura prehispánica en el sur occidente de Colombia. Conferencia en el Programa doctorado interuniversitario de Ciencias ambientales. Universidad del Valle Colombia. 